women without oestrogen replacement therapy should have a daily calcium intake of $1500 \mathrm{mg} / \mathrm{d}$. Only a small fraction of the postmenopausal women in our sample had a daily calcium intake at this level. However, the FFQ used in this study has been validated, and a tendency has been found towards underestimation of both calcium and energy intake with this FFQ compared with the four, one week dietary records that were used as a reference method in a validation study among 129 women (unpublished data). On average, calcium intake was underestimated by $67 \mathrm{mg} / \mathrm{d}(8 \%)$ and total energy intake by $345 \mathrm{kcal} / \mathrm{d}(20 \%)$. Despite this possible slight underestimation of calcium intake, if Sweden were to adopt the US recommendation, most postmenopausal women would have to be advised to increase their calcium intake by supplementation, or food fortification would have to be introduced since it is difficult to obtain $1500 \mathrm{mg} / \mathrm{d}$ of calcium from the diet. ${ }^{4}$

Even though some of the variables examined in our study were statistically associated with calcium intake, the absolute differences in calcium intake between subcategories of the variables were small. Thus none of the variables analysed in our study can be used for targeting health information on an increase in calcium intake in subgroups of the population.

* The study group of Multiple Risk Survey on Swedish Women for Eating Assessment (MRS SWEA); H O Adami, L Bergkvist, A Bruce, J Leppert, G Nilsson, I Ringqvist, B Vessby.

1 Schaafsma G. The scientific basis of recommended dietary allowances for calcium. F Intern Med 1992;231:187-94 2 NIH Consensus Conference. Optimal calcium intake. $\mathscr{f} A M A$ 1994;272:1942-8.

3 Hegsted DM. Calcium and osteoporosis. 7 Nutr 1986;116: 2316-9.

4 Harward MP. Nutritive therapies for osteoporosis. Clin Nutr $1993 ; 77: 889-98$

\title{
Helicobacter pylori seropositivity and cardiovascular risk factors in a multicultural workforce
}

\author{
R K R Scragg, A Fraser, P A Metcalf
}

Recent epidemiological studies suggest a positive association between Helicobacter pylori infection and coronary heart disease. Coronary heart disease cases are more likely to be $H$ pylori seropositive than controls, ${ }^{12}$ while controls who are $H$ pylori seropositive have higher fibrinogen ${ }^{23}$ and lower high density lipoprotein (HDL) cholesterol ${ }^{3}$ concentrations than seronegative subjects. In view of these reports, we have examined whether $H$ pylori is associated with the major cardiovascular risk factors in a work based sample in New Zealand, where both $H$ pylori infection and coronary heart disease are common.

Methods: Study subjects came from a cross sectional survey of 5677 participants aged 40-64 years, carried out at worksites in New

Departments of Community Health R K R Scragg P A Metcalf

and Medicine A Fraser

University of Auckland, Private Bag, Auckland, New Zealand

Correspondence to: Dr Robert Scragg. Accepted for publication May 1996

f Epidemiol Commun Health 1996;50:578-79
Zealand during 1988-90. Full details on sampling, response rate of $67 \%$, and methods have been described. ${ }^{4}$ Briefly, participants fasted overnight before presenting for glucose tolerance tests. During this, they answered questions on ethnicity, occupation (which was used to determine their socioeconomic status by the Elley-Irving criteria; cited in reference 4), and weekly leisure time physical activities in the last three months. The latter was defined as vigorous if it made participants short of breath, and otherwise moderate.

Blood pressure was measured twice with a random zero mercury sphygmomanometer and averaged. Commercial methods were used on fasting serum samples to measure total cholesterol and triglyceride on a Chem I analyser (Technicon, Tarrytown, NY), HDL cholesterol on a Cobas Fara centrifugal analyser (Boehr-

Table 1 Comparison of mean cardiovascular risk factor concentrations, adjusted for age, ethnic group, and socioeconomic status, between participants positive and negative for Helicobacter pylori infection

\begin{tabular}{lccc}
\hline Cardiovascular risk factor & $\begin{array}{l}\text { H pylori positive } \\
\text { Mean }(S E) \\
(n=322)\end{array}$ & $\begin{array}{l}\text { H pylori negative } \\
\text { Mean (SE) } \\
(n=257)\end{array}$ & $\begin{array}{l}\text { Mean difference } \\
(95 \% \text { CI })\end{array}$ \\
\hline Blood pressure (mmHg) & & & \\
Systolic & $126.0(0.8)$ & $126.7(0.9)$ & $-0.7(-3.0,1.6)$ \\
Diastolic & $78.9(0.6)$ & $79.6(0.7)$ & $-0.8(-2.6,1.2)$ \\
Total cholesterol (mmol/1) & $6.20(0.07)$ & $6.08(0.08)$ & $0.12(-0.09,0.32)$ \\
HDL cholesterol (mmol/l) & $1.20(0.02)$ & $1.27(0.02)$ & $-0.06(-0.12,-0.01)$ \\
Triglycerides (mmol/l) & $1.71(0.07)$ & $1.62(0.08)$ & $0.09(-0.11,0.29)$ \\
Fasting glucose (mmol/l) & $5.51(0.04)$ & $5.47(0.05)$ & $0.03(-0.09,0.15)$ \\
\hline
\end{tabular}


inger, Mannheim), and plasma glucose (Roche Products, NZ).

Six hundred participants (200 from each ethnic group) without diabetes were randomly selected. Of these, 579 (195 Maori, 194 Pacific Islanders, 190 Europeans) had stored serum aliquots (at $-80^{\circ} \mathrm{C}$ ) that were retrieved and tested for $H$ pylori antibody response by enzyme immunoassay (Roche Diagnostics). This has been validated in an Auckland outpatient sample against a rapid urease test (sensitivity $96 \%$, specificity $67 \%$ ) and a ${ }^{13} \mathrm{C}$ urea breath test (sensitivity $91 \%$, specificity $82 \%$ ). ${ }^{5}$ The Cochran-Mantel-Haenszel statistic was used to compare adjusted $H$ pylori seropositive proportions, and adjusted means were calculated using $S A S$ (SAS Institute, Cary, NC). There was no design effect $(\mathrm{d}=0.79)$ due to clusteredsampling; ${ }^{6}$ therefore, the effect of workplace variation has not been controlled for in analyses.

Results: The proportion of subjects seropositive for $H$ pylori varied with ethnic group (Pacific Islanders 73.2\%, Maori 57.4\%, Europeans $35.8 \%, \mathrm{p}<0.001$ ); increased with age (40-49 years $48.0 \%, 50-64$ years $63.4 \%, \mathrm{p}=$ $<0.001)$; but was similar for men and women $(55.6 \%$ and $55.7 \%$, respectively, $\mathrm{p}=0.98)$. Adjusting for age and ethnicity, $H$ pylori seropositivity did not vary significantly: with socioeconomic status being $49.7 \%$ in the highest groups 1 and 2 compared with group 3 $(52.8 \%, \mathrm{p}=0.36)$, group $4(60.6 \%, \mathrm{p}=0.06)$ and groups 5 and $6(56.6 \%, p=0.30)$; nor did it vary significantly between participants doing vigorous leisure time activities $(54.9 \%, \mathrm{p}=$ 0.09 ) or moderate activities $(54.3 \%, \mathrm{p}=0.26)$ compared with inactive participants $(60.9 \%)$.

Adjusted mean levels of blood pressure, total cholesterol, fasting triglycerides, and fasting glucose were similar for seropositive and seronegative groups (table 1). In contrast, seropositive participants had a lower adjusted mean HDL cholesterol level compared with those who were seronegative $(p=0.029)$.

Discussion: We have found that $H$ pylori seropositivity is inversely related to HDL cholesterol concentrations, but unrelated to other major cardiovascular risk factors. The specificity of our test indicates the presence of falsepositives in our sample; this disease misclassification, which is likely to be non-differential, suggests that we have underestimated the true HDL cholesterol difference between people with and without $H$ pylori. The results from our work based sample may not apply to unemployed populations which include people too sick to work. The decreased HDL cholesterol concentration in participants seropositive for $H$ pylori could be due to chance, although this finding is consistent with a previous report. ${ }^{3}$ Our results suggest that if $H$ pylori is a risk factor for coronary heart disease, it may operate through a mechanism involving HDL cholesterol. This finding is potentially important as $H$ pylori infection is readily treatable, although there are no data on HDL cholesterol concentrations after eradication of the infection.

1 Mendall MA, Goggin PM, Molineaux N, et al. Relation of Helicobacter pylori infection and coronary heart disease. Br Heart f 1994;71:437-9.

2 Patel P, Mendall MA, Carrington D, et al. Association of Helicobacter pylori and Chlamydia pneumoniae infections with coronary heart disease and cardiovascular risk factors. $B M F$ 1995;311:711-4.

3 Murray LJ, Bamford KB, O'Reilly DPJ, McCrum EE, Evans $\mathrm{AE}$. Helicobacter pylori infection: relation with cardioAE. Helicobacter pylori infection: relation with cardio-
vascular risk factors, ischaemic heart disease and social vascular risk factors, ischaemic heart disease
class. Gut 1995;37(suppl 1):A11 (abstract 41).

4 Scragg R, Baker J, Metcalf P, Dryson E. Prevalence of diabetes mellitus and impaired glucose tolerance in a New Zealand mellitus and impaired glucose tolerance in a New Z
multiracial workforce. NZ Med $\mathcal{F} 1991 ; 104: 395-7$.

5 Fraser AG, Ali MR, McCullough S, Haystead A. Diagnostic tests for Helicobacter pylori - can they help select patients for endoscopy? NZ Med $\mathcal{F}$ 1996;109:95-8.

6 Rao JNK, Scott AJ. A simple method for the analysis of clustered binary data. Biometrics 1992;48:577-585. 